\title{
Psychiatric Comorbidities in Adolescents with Obesity: A Wake-Up Call for Life Course and Multisectoral Interventions
}

\author{
Bhavneet Bharti ${ }^{1} \cdot$ Prahbhjot Malhi $^{2}$
}

Received: 15 January 2021 / Accepted: 15 January 2021 / Published online: 4 February 2021

(C) Dr. K C Chaudhuri Foundation 2021

Obesity in children is the emerging medical bellwether of compromised lifestyle, poor dietary habits, and stressed behavior. Given the easy access to junk food and sedentary lifestyle among the youth in modern-day society, high body mass index (BMI) threatens to impact health and longevity. In a meta-analysis, obese children and adolescents were nearly five times more likely to be obese in their adulthood years relative to those who were not obese [1]. Indeed, studies that utilize a life course approach to obesity have documented that high BMI during adolescence is associated with several short- and long-term medical and psychosocial comorbidities. Some of the mental health problems associated with obesity include low self-esteem, increased body dissatisfaction, increased risk of psychiatric disorders, particularly depression [2]. The study by Arumuganathan et al. in the current issue has explored an important and often ignored issue and highlighted the body image issues and psychiatric comorbidities among the obese Indian school-going adolescents [3]. The study found significantly higher rates of social phobia (36\%), followed by specific phobia (19\%), and major depressive disorder (19\%) among the obese adolescents. Body image issues were more salient for the obese as compared to the normal matched controls $(90 \%$ vs. $29 \%$, respectively). Despite the significance of their findings, the study design cannot rule out reverse causality as psychiatric disorders are known to precipitate obesity among adolescents. For example, a meta-analysis of 13 longitudinal studies that assessed the bidirectional relationship between obesity and depression showed that depressed adolescents had $70 \%$ higher risk of obesity whereas obese adolescents had $40 \%$ higher risk of depression with absolute risk difference of depression

Bhavneet Bharti

bhavneetsahul@gmail.com

1 Dr BR Ambedkar Institute of Medical Sciences, Sahibzada Ajit Singh Nagar, Punjab, India

2 Department of Pediatrics, Post Graduate Institute of Medical Education and Research, Chandigarh, India leading to adolescent obesity $3 \%$ higher than obesity leading to depression [4]. Given this bidirectional relationship between obesity and psychiatric problems and the recent evidence of interaction between adverse childhood experiences and obesity, there is urgent need for multisectoral approach for management of obese adolescents. Obesity is unfortunately also associated with considerable weight stigmatization and discrimination leading to social isolation which could be major barrier to behavior change and there is a need for sensitive public health campaigns. Moreover, research highlights overlapping genetic susceptibility and neuroendocrine processes between obesity and psychiatric disorders. Nearly $12 \%$ of the genetic component of depression has been found to be shared with obesity [5].

Limited evidence is available regarding the most effective treatments for obesity in adolescents with psychiatric disorders and health care professionals struggle to seek effective strategies to address this global epidemic. Most interventions do not address the rampant social consequences of obesity such as body shaming, peer victimization, stigmatization, and discrimination. Clearly then, a deterministic nutritional approach alone will be suboptimal in dealing with this global health emergency that is associated with wide ranging ramifications for the physical and mental health of adolescents. There is a need for life course, multisectoral, and holistic approach in dealing with obesity among adolescents, particularly in view of the high rate of associated psychiatric comorbidities. Indeed, the role of early mental health evaluation and intervention is undeniably vital.

\section{Compliance with Ethical Standards}

Conflict of Interest None.

\section{References}

1. Simmonds M, Llewellyn A, Owen CG, Woolacott N. Predicting adult obesity from childhood obesity: a systematic review and meta-analysis. Obes Rev. 2016;17:95-107. 
2. Styne DM, Arslanian SA, Connor EL, et al. Pediatric obesity-assessment, treatment, and prevention: an endocrine society clinical practice guideline. J Clin Endocrinol Metab. 2017;102:709-57.

3. Arumuganathan S, Sagar R, Mehta M, Ilango TS. Psychiatric comorbidities and body shape dissatisfaction in adolescents with obesity - a school-based case-controlled study. Indian J Pediatr. 2020. https://doi.org/10.1007/s12098-020-03367-z.

4. Mannan M, Mamun A, Doi S, Clavarino A. Prospective associations between depression and obesity for adolescent males and females- a systematic review and meta-analysis of longitudinal studies. PLoS One. 2016;11:e0157240

5. Afari N, Noonan C, Goldberg J, et al. Depression and obesity: do shared genes explain the relationship? Depress Anxiety. 2010;27: 799-806.

Publisher's Note Springer Nature remains neutral with regard to jurisdictional claims in published maps and institutional affiliations. 